\title{
Causal Laws are ObJectifications of INDUCTIVE SCHEMES*
}

\author{
Wolfgang Spohn \\ Fachbereich Philosophy \\ Universität Konstanz \\ 78457 Konstanz \\ Germany
}

And this paper is an attempt to say precisely how, thus addressing a philosophical problem which is commonly taken to be a serious one. It does so, however, in quite an idiosyncratic way. It is based on the account of inductive schemes I have given in (1988) and (1990a) and on the conception of causation I have presented in (1980), (1983), and (1990b), and it intends to fill one of many gaps which have been left by these papers.

Still, I have tried to make this paper self-contained. Section 1 explains the philosophical question this paper is about; in more general terms it asks what might be meant by objectifying epistemic states or features of them and to which extent epistemic states can be objectified. The next sections introduce the basis I rely on with formal precision and some explanation; section 2 deals with induction and section 3 with causation. Within these confines, section 4 attempts to give an explication of the relevant sense of objectification and section 5 investigates the extent to which various features of epistemic states are objectifiable. The two most salient results are roughly that the relation " $A$ is a reason for $B$ " cannot be objectified at all and that the relation " $A$ is a cause of $B$ " can be objectified only under substantial, though reasonable restrictions.

What has all of this to do with probability? A lot. The paper trades on a pervasive duality between probabilistic and deterministic epistemology, between a probabilistic representation of epistemic states together with a theory of probabilistic causation and another representation of epistemic states which I call deterministic because it lends itself, in a perfectly parallel fashion, to a theory of de-

\footnotetext{
* Published in Jacques-Paul Dubucs (ed.), Philosophy of Probability, Kluwer, Dordrecht 1993, pp. 223-265.
} 
terministic causation. ${ }^{1}$ Here I explicitly deal only with the deterministic side, but the duality should pave the way for further conclusions concerning objective probabilities and statistical laws. This outlook is briefly expanded in the final section 6.

\section{Is Causation Objective?}

Objectivity has many different facets which call for many different explanations. One facet is truth. We think that what is true is objectively true, independent from any subjective point of view. In this sense it is an open issue whether causation is objective, whether causal statements are (objectively) true or false. The common intuition is affirmative, but it's not easy to philosophically account for it.

The issue initiates with David Hume. Indeed, it hides right in his two definitions of causation as what he calls a philosophical and a natural relation. ${ }^{2}$ Causation as a philosophical relation is constituted by precedence, contiguity, and regularity; it is objective because precedence, contiguity, and the existence of a suitable regularity are objective matters. Whereas causation as a natural relation is constituted by precedence, contiguity, and association (of the effect with the cause in the mind of some epistemic subject); it is not objective because on this view causal statements as such are neither true nor false, but depend on the epistemic state of the subject. ${ }^{3}$ It is an intricate exegetical issue precisely how Hume understands the relation between his two definitions. ${ }^{4}$ The most plausible view is, roughly, that the associationist theory is conceptually more basic and is provided with an explanation by the regularity theory because it is the regularities which, to a large extent, shape our associations. ${ }^{5}$ However, Hume is not free from ambiguity; in his response to the charge of an imagined realist that his notion of causation is not objective he quickly resorts from the associationist to the regularity

\footnotetext{
${ }^{1}$ I have more fully presented this duality in (1983) and (1988), sect. 7.

2 Cf. Hume (1739), pp. 170ff.

3 Thus, a statement of the form " $A$ is a cause of $B$ relative to the subject $X$ " may well be objective and objectively true; relativization yields objectification (cf. Mühlhölzer, 1988). But of course, we are interested in the truth of the unrelativized statement, and it would certainly be inappropriate to get rid of the relativization simply by existential quantification.

4 Cf. Mackie (1974), ch. 1, and Beauchamp, Rosenberg (1981), ch. 1, for two thorough discussions.

5 That is the line of thought Beauchamp, Rosenberg (1981), ch. 1, end up with - plausibly in my view.
} 
theory. ${ }^{6}$

Since then the problem stays with us; and the ways sought to get out of it are too numerous to be counted here. I mention only some of them:

One may deny the problem by giving an outright objectivist account of causation. One may conceive of causation as a kind of physical ingredient of the world, e.g. as energy transfer, as is often thought. ${ }^{7}$ Or one may conceive of it as an objective structural feature of the world constituted by laws of nature (this is the most popular view ${ }^{8}$ ), by a relation of counterfactuality (as has been urged in our days in particular by Lewis 1973), or as a certain second order universal (an Australian proposal ${ }^{9}$ ). But I remain skeptical: because there is a need to explain the most prominent and peculiar epistemological role of the notion of causation rightly emphasized by Hume, because it seems that this explanation cannot simply be given in terms of the subject's grasp of how causation objectively is, and because it is hard to see which other kind of explanation is available to purely objectivistic accounts of causation - though this is not the place to argue this point. ${ }^{10}$

Or one may deny the problem by acquiescing in an epistemologically relativized notion of causation and talking us out of our realistic intuition. This line is most prominently pursued today by Putnam (cf., e.g., his 1983) and, in quite a different way, also by van Fraassen. ${ }^{11}$ But this subjectivistic strategy can at most succeed, if it does not only try to make us believe that the realistic intuition concerning causation is a confusion or an illusion, but offers us a plausible account or a convincing substitute for it.

So, there is no way of avoiding to face the problem. Facing the problem means trying to integrate the two one-sided positions, that is, to give both an objectivistic and subjectivistic account of causation and to specify their relation. If it is true that this relation does not simply consist in the subject's grasp of objective causation, then the direction of analysis should presumably be reversed, i.e. the objec-

\footnotetext{
6 Cf. Hume (1739), pp.167-169.

7 Cf., e.g., Aronson (1971) and Fair (1979).

8 To be found in many places; see, e.g., Hempel (1965), pp. 348ff., and Carnap (1966), ch. 19. Of course, this popular view runs into the well-known difficulty of characterizing laws of nature, i.e. of specifying a criterion of lawlikeness, without recourse to causation.

9 Cf. in particular Tooley (1987), sect. 8.3.

10 The most extensive recent criticism of objectivistic or realistic approaches to laws of nature and related things may be found in van Fraassen (1989), part I.

11 Cf. his (1980), pp. 112ff., where he argues the theory of causation to be almost wholly absorbed by a theory of explanation which can be understood only in a subjectively or pragmatically relativized way; only an empty objective characterization of "the causal net $=$ whatever structure of relations science describes" (p.124) remains.
} 
tivistic account should be understood as some kind of objectification of the subjectivistic one.

There are not so many models for doing this. One may indulge into Kant's complicated doctrine of transcendental idealism in his Kritik der reinen Vernunft in which the present objectification problem is meshed with other and, in the Kantian context, more salient ones concerning space, time, the self, and other objects. In modern times the awareness that the subjectivist and the objectivist side need to be mediated is still lively; and Salmon (1984) is certainly one of the most forceful attempts to meet this need, i.e. to defend an, as he calls it, ontic conception of causation without losing the virtues of an epistemic conception. However, I am not sure how to categorize this and other recent attempts as objectifications of a subjectivistic account.

In a way, Hume himself may be said to have offered a solution of the problem. As already mentioned, one may take causation as basically non-objective as specified in his associationist theory of causation, and one may then objectify it to the extent to which our associations can be explained or supported by existing regularities; insofar our associations do not have such an objective basis, causation is not objectifiable.

Isn't this good enough a solution? No, because the associationist theory isn't good enough. There are various well-known problems in the logic of causation it cannot cope with. Among them, the basic problem is that it cannot distinguish between the causes of an effect and mere symptoms or indicators preceding it. ${ }^{12}$ I propose a simple remedy: improve the associationist theory and then adapt the account of objectification.

The improvement consists of two steps. Since for Hume induction is more or less synonymous to association - the inductively inferred beliefs are those associated with other beliefs -, the first step is to give a general and precise account of a subject's inductions or associations; this is the intent of the theory of inductive schemes explicated as so-called natural conditional functions (NCFs) in the next section. The second step, then, is to reconstruct a Humean theory of causation on that improved subjective basis; this will be undertaken also in section 3 as far as it will be required.

Afterwards, we can turn to the question how this subjectively relativized theory of causation can be objectified. In fact, I propose to investigate a more general question: An inductive scheme, or a NCF, characterizes the epistemic state of

12 Cf., e.g., Mackie (1974), pp. 81ff. 
some subject. Such an epistemic state has various features. It includes a specific causal picture, for instance, or it contains specific beliefs. These features are sometimes a matter of truth and falsity and sometimes not. For example, beliefs can certainly be true or false, whereas a subjective probability for some contingent proposition cannot sensibly be called true or false; it can only be welladvised or ill-guided. ${ }^{13}$ So we need a general explanation of what it means to make such a feature a matter of truth and falsity. This allows us to pose the question of objectification for each feature of an epistemic state, namely as the question to which extent that feature can be made a matter of truth and falsity. Section 4 attempts to give that explanation and section 5 attempts to answer the question of objectification for some features of an epistemic state, among them its causal picture as explicated in section 3 .

\section{Induction}

What might a theory of induction be expected to yield? No more and no less, I think, than a dynamic account of epistemic states which specifies not only their static laws, but also their laws of change - where these laws are most plausibly understood as laws of rationality. ${ }^{14}$ The forms these laws take depend, of course, on how epistemic states are represented. The axioms of mathematical probability are the static laws of a probabilistic representation, and the principle of maximizing relative entropy as well as various rules of conditionalization are its most plausible candidates for dynamic laws. Plain belief which affirms or denies a proposition or does neither and thus admits only of three grades ${ }^{15}$ is most easily represented by a set of propositions, namely those held to be true. The most plausible static laws are that such a set be consistent and deductively closed. However, there is no general dynamic account of epistemic states represented in this way. Even if one returns to the probabilistic representation and equates plain belief with subjective probability 1 , one does not arrive at a general dynamic theory of plain belief because all standard probabilistic laws of change do not allow what must be allowed, namely to retract from probability 1 (whatever has probability 1 , keeps it according to these laws) and thus to give up plain beliefs. Hence, a dif-

13 Even if the subjective probability matches the objective probability of that proposition, should it have one, it would be inappropiate to call it true.

14 In my (1993) I have tried to characterize the role of laws of rationality.

15 This is how I want "plain belief" to be understood. It is the kind of belief all epistemic logics are about. 
ferent representation of epistemic states and a different theory is required in order to account for the dynamics of plain belief. In (1988) I have presented such a theory and explained its details and the drawbacks of rival theories. ${ }^{16}$ Here, I have to restrict myself to briefly presenting its formal structure.

Throughout, I shall make the convenient assumption that propositions construed as set of possible worlds serve as objects of belief and as objects of causation as well. This is problematic in various ways ${ }^{17}$; but I shall not bother with these problems because they do not essentially affect the present issue. Thus, let $\Omega$ denote a set of possible worlds, as philosophers say, or a sample space, as probability theorists say, i.e. just an exhaustive set of mutually exclusive possibilities; there is no need of further clarifying the nature of these possibilities. Elements of $\Omega$ will be denoted by $\omega, v, \alpha$, etc. Not worrying about questions of measurability, we take each subset of $\Omega$ to represent a proposition; propositions are denoted by $A, B, C, D, E$, etc. The basic concept, then, is formally very simple; it is given by

Definition 1: $\kappa$ is a natural conditional function (a $N C F$ ) iff $\kappa$ is a function from $\Omega$ into the set of natural numbers such that $\kappa^{-1}(0) \neq \varnothing$. A NCF $\kappa$ is extended to propositions by defining $\kappa(A)=\min \{\kappa(\omega) \mid \omega \in A\}$ for each $A \neq \varnothing$ and $\kappa(\varnothing)=$ $\infty .18$

A NCF $\kappa$ is to be interpreted as a grading of disbelief. If $\kappa(\omega)=0$, then $\omega$ is not disbelieved, i.e. $\omega$ might be the actual world according to $\kappa$. Because not every world can be denied to be the actual one, Definition 1 requires that $\kappa(\omega)=0$ for some $\omega \in \Omega$. If $\kappa(\omega)=n>0$, then $\omega$ is disbelieved with degree $n$. A proposition is then assigned the minimal degree of disbelief of its members. Thus, if $\kappa(A)$ $=n>0$, then $A$ is disbelieved with degree $n$. And if $\kappa(A)=0$, then $A$ is not disbelieved, i.e. $A$ might be true according to $\kappa$. However, $\kappa(A)=0$ does not mean that

16 In particular, I there explain in which way the theory of NCFs generalizes the theory by which it was most heavily influenced, namely the somewhat restricted account of belief change developed by Gärdenfors and his collaborators (and most extensively presented in Gärdenfors, 1988). Hansson (1991) generalizes this account in more Gärdenforsian terms.

17 For example, it seems that one must take sets of centered instead of uncentered possible worlds in order to account for indexical belief. Also, one might argue that the identification of the objects of belief and those of the causal relation is guilty of a confusion of metaphysical and epistemic modality. And these are only two of many problems.

18 In my (1988), I have defined so-called ordinal conditional functions which are a bit more general (and a bit more awkward) in taking ordinal numbers as values. This generality will not be required here. 
$A$ is believed according to $\kappa$. Belief in $A$ is rather expressed by disbelief in $\sim A^{19}$, i.e. by $\kappa(\sim A)>0$ or $\kappa^{-1}(0) \subseteq A$. I call $\kappa^{-1}(0)$ the net content of the epistemic state $\kappa$. Thus, all and only the supersets of the net content of $\kappa$ are plainly believed in $\kappa$, i.e. held to be true. This implies that plain belief is consistent and deductively closed; but these features go hand in hand with the assumption that the objects of beliefs are propositions.

Two simple, but important properties of NCFs immediately follow: the law of negation that for each proposition $A$ either $\kappa(A)=0$ or $\kappa(\sim A)=0$ or both, and the law of disjunction that for all propositions $A$ and $B, \kappa(A \cup B)=\min (\kappa(A), \kappa(B))$.

According to a NCF $\kappa$, propositions are believed in various degrees. It is useful to explicitly introduce the function expressing these degrees, because it is more vivid than the above disbelief talk:

Definition 2: $\beta$ is the belief function associated with the NCF $\kappa$ iff, for each subset $A$ of $\Omega, \beta(A)=\kappa(\sim A)-\kappa(A)$; and $\beta$ is a belief function iff it is associated with some NCF. 20

Thus, $\beta(\sim A)=-\beta(A)$, and $A$ is believed true or false or neither according to $\beta$ (or $\kappa)$ depending on whether $\beta(A)>0$ or $<0$ or $=0$. However, there is no simple law for disjunctions in terms of belief functions; this is why NCFs are preferable on the formal score.

So far, the various degrees of belief did not really play a theoretical role. But they are crucial for a dynamic account of plain belief. The central notion is specified in

Definition 3: Let $\kappa$ be a NCF and $A$ a non-empty proposition. Then the A-part of $\kappa$ is the function $\kappa(. \mid A)$ defined on $A$ by $\kappa(\omega \mid A)=\kappa(\omega)-\kappa(A)$ for each $\omega \in A$. Again, this function is extended to all propositions by setting $\kappa(B \mid A)=\min \{\kappa(\omega$ | $A) \mid \omega \in A \cap B\}=\kappa(A \cap B)-\kappa(A)$ for each $B \subseteq \Omega$. Finally, if $\beta$ is the belief function associated with $\kappa$, we define, as in Definition 2, $\beta(B \mid A)=\kappa(\sim B \mid A)$ $\kappa(B \mid A)$.

Definition 3 is tantamount to the law of conjunction that $\kappa(A \cap B)=\kappa(A)+\kappa(B$ I $A$ ) for all propositions $A$ and $B$ with $A \neq \varnothing$.

19 “ " is used here to denote the set-theoretical complement (with respect to $\Omega$ ).

20 Shenoy (1989) has convinced me of the usefulness of explicitly introducing this concept, and the brief definition is due to Bernard Walliser. 
The $A$-part $\kappa(. \mid A)$ of $\kappa$ can be viewed as a NCF with respect to the restricted possibility space $A$ and thus as a grading of disbelief conditional on A. Accordingly, $\beta(. \mid A)$ expresses degrees of belief conditional on $A$.

It is obvious that a NCF $\kappa$ is uniquely determined by its $A$-part $\kappa(. \mid A)$, its $\sim A$ part $\kappa(. \mathrm{I} \sim A)$, and the degree $\beta(A)$ of belief in $A$. This marks an important point of difference to various other approaches which in effect operate only with orderings and not with gradings of disbelief. Within these approaches one may perhaps also define $A$-parts, i.e. orderings of disbelief conditional on $A$. But there is no way to uniquely combine various parts of an ordering, and therefore the following ideas cannot be carried over to orderings of disbelief.

This uniqueness suggests a simple model of belief revision for NCFs. If a piece of information consists only in the proposition $A$, then it is plausible to assume that only the old degree $\beta(A)$ of belief in $A$ gets changed to some new degree $\beta^{\prime}(A)=n$, whereas the $A$-part and the $\sim A$-part of the old NCF $\kappa$ are left unchanged; $n, \kappa(. \mid A)$, and $\kappa(. \mid \sim A)$ then determine a new NCF $\kappa^{\prime}$ (and a new belief function $\beta^{\prime}$ ), which I call the $A, n$-conditionalization of $\kappa$. There are also more complicated models in which the information need not concern a single proposition. But these remarks already indicate that a full dynamics of plain belief and thus a full theory of induction can be stated in terms of NCFs.

An account of conditionalization immediately yields the epistemologically important notions of dependence and independence. Two propositions are independent iff conditionalization with respect to the one does not affect the epistemic status of the other. Formally:

Definition 4: Let $\beta$ be the belief function associated with the NCF $\kappa$, and $A, B$, and $C$ three non-empty propositions. Then $A$ and $B$ are independent with respect to $\beta$ (or $\kappa$ ) iff $\beta(B \mid A)=\beta(B \mid \sim A)$, i.e. iff $\kappa\left(A^{\prime} \cap B^{\prime}\right)=\kappa\left(A^{\prime}\right)+\kappa\left(B^{\prime}\right)$ for each $A^{\prime}$ $\in\{A, \sim A\}, B^{\prime} \in\{B, \sim B\}$; and they are independent conditional on $C$ w.r.t. $\beta$ (or $\kappa$ ) iff $\beta(B \mid A \cap C)=\beta(B \mid \sim A \cap C)$.

Of course, (conditional) independence may be generalized to a relation between whole algebras of propositions, and so forth. Indeed, the fact that conditional dependence and independence with respect to belief functions behave precisely like their probabilistic counterparts is the technical reason why NCFs will form a suitable base for a parallel theory of deterministic causation.

A closely related and equally important notion is the concept of a reason. Being a reason is always relative to an epistemic state, and given such a state a rea- 
son strengthens the belief in, or, in other words, is positively relevant to, what it is a reason for. Formally:

Definition 5: Let $\beta$ be the belief function associated with the NCF $\kappa$, and $A, B$, and $C$ three propositions. Then $A$ is a reason for $B$ relative to $\beta$ or $\kappa$ iff $\beta(B \mid A)>$ $\beta(B \mid \sim A)$; and $A$ is a reason for $B$ conditional on $C$ relative to $\beta$ or $\kappa$ iff $\beta(B \mid A \cap$ $C)>\beta(B \mid \sim A \cap C)$.

According to this definition, the relation of being a reason is symmetric, but not transitive, in analogy to probabilistic positive relevance, but in sharp contrast to the narrower relation of being a deductive reason (which is just set inclusion between contingent propositions). ${ }^{21}$ Moreover, being a reason does not presuppose that the reason is actually given, i.e. believed; on the contrary, whether $A$ is a reason for $B$ relative to $\beta$ is independent of the degree $\beta(A)$ of belief in $A$.

Since the value 0 has the special role of a dividing line between belief and disbelief, different kinds of reasons can be distinguished:

\section{Definition 6:}

$A$ is a $\left\{\begin{array}{c}\text { additional } \\ \text { sufficient } \\ \text { necessary } \\ \text { weak }\end{array}\right\}$ reason for $B$ w.r.t. $\beta$ or $\kappa$ iff $\left\{\begin{array}{l}\beta(B \mid A)>\beta(B \mid \sim A)>0 \\ \beta(B \mid A)>0 \geq \beta(B \mid \sim A) \\ \beta(B \mid A) \geq 0>\beta(B \mid \sim A) \\ 0>\beta(B \mid A)>\beta(B \mid \sim A)\end{array}\right\}$.

Conditional reasons of the various kinds are defined similarly. If $A$ is a reason for $B$, it belongs at least to one of these four kinds; and there is just one way of belonging to several of these kinds, namely by being a necessary and sufficient reason. Sufficient and necessary reasons are the more important ones; but additional and weak reasons, which do not show up in plain belief and are therefore usually neglected, well deserve to be allowed for by Definition 6 .

No further development of the theory of NCFs as a substitute of Hume's theory of association will be needed here. So, the next step in amending Hume is to give an account of causation relative to the NCFs.

21 This structural fact most clearly shows that deductive logic may, in a way, have been misleading as a model of human reasoning. 


\section{Causation}

This account will be quite brief because I have more thoroughly dealt with the matter in the papers referred to.

The first thing to do is to give possible worlds a temporal structure: Let $I$ be a non-empty set of factors or variables; we may assume $I$ to be finite in order to avoid all the technical problems related to infinity. Each variable $i \in I$ is associated with a set $\Omega_{i}$ containing at least two members; $\Omega_{i}$ is the set of values $i$ may take. The set $\Omega$ of possible worlds is then represented as the Cartesian product of all the $\Omega_{i}(i \in I)$; hence, each $\omega \in \Omega$ is a possible course of events, a function assigning to each variable $i \in I$ the value $\omega(i)$ taken by $i$ in the possible world $\omega$.

Since metric properties of time are irrelevant, time may then simply be represented by a weak, i.e. transitive and connected order $\leq$ on the set $I$ of variables; < denotes the corresponding irreflexive order on $I$. and $i \approx j$ is to say that the variables $I$ and $j$ are simultaneous. Discreteness of time is implied by the finiteness of $I$. But even if $I$ were infinite, one should assume that time is discrete and treat continuous time as the limit of ever finer discrete time (as it is done in the theory of stochastic processes).

Time should be associated not only with variables, but, if possible, also with propositions. Therefore a proposition $A$ is defined to be a $J$-measurable or, in short, a $J$-proposition for a set $J \subseteq I$ of variables iff for all $v, \omega \in \Omega$ agreeing on $J$, i.e. with $\cup(i)=\omega(i)$ for all $i \in J, \cup \in A$ iff $\omega \in A$; intuitively one might say that a $J$-proposition is only about the variables in $J$ and does not say anything about other variables. There are many contingent propositions which are about single variables, and the temporal order of variables is easily carried over to them. Indeed, I see no loss in restricting causes and effects to be such, so to speak, logically simple propositions which are about one variable only.

The general idea of the explication of causation is now a common one: $A$ is a cause of $B$ in the world $\omega$ iff $A$ and $B$ obtain in $\omega, A$ precedes $B$, and $A$ raises the epistemic or metaphysical status of $B$ under the circumstances obtaining in $\omega$. This characterization fits many prominent conceptions of causation the main difference of which lies in their account of what I have called the epistemic or metaphysical status and which have a minor difference over whether they should take precedence strictly on loosely so as to include simultaneity. Here, the condition that $A$ and $B$ obtain in $\omega$ may be expressed by $\omega \in A \cap B$. The condition that $A$ precedes $B$ in the strict sense translates into the condition that for some $i, j \in \omega A$ 
is an $i$-proposition, $B$ a $j$-proposition, and $i<j$; however, I shall only require that $i$ $\leq j$, that is, I take precedence in the loose sense including simultaneity. ${ }^{22}$ The epistemic status of $B$ is specified, of course, by a belief function $\beta$, which is raised by $A$ iff it is higher conditional on $A$ than conditional on $\sim A$. Finally, it may be argued that in the case of direct causation, the circumstances obtaining in $\omega$ may be identified with the whole past of the direct effect in $\omega$ with the exception of the direct cause - where a loose sense of precedence including simultaneity goes hand in hand with a loose sense of past including presence. Thus I define: If $A$ is an $i$-proposition, $B$ a $j$-proposition, and $i \leq j$, then the circumstances of $A$ 's directly causing $B$ in $\omega$ are defined as $C_{A, B, \omega}=\{v \mid v(k)=\omega(k)$ for all $k \in I$ with $k$ $\leq j$ and $k \neq i, j\}$, i.e. as the past and presence of $B$ in $\omega$ with the exception of $A$ and $B$. Thus we get:

Definition 7: Let $\omega \in \Omega, i, j \in I, A$ be a $i$-proposition, and $B$ a $j$-proposition. Then $A$ is a direct cause of $B$ in $\omega$ relative to the belief function $\beta$ or the associated NCF $\kappa$ iff $\omega \in A \cap B, i \leq j$, and $\beta\left(B \mid A \cap C_{A, B, \omega}\right)>\beta\left(B \mid \sim A \cap C_{A, B, \omega}\right)$, i.e. $A$ is a reason for $B$ conditional on $C_{A, B, \omega}$ relative to $\beta$ or $\kappa$. And $A$ is called an additional, sufficient, necessary, or weak direct cause of $B$ in $\omega$ according to whether $A$ is an additional, sufficient, necessary, or weak reason for $B$ conditional on $C_{A, B, \omega}$.

In my (1980), (1983), and (1990b) I have more fully argued for the adequacy of that definition. ${ }^{23}$ And in my (1990b) I have also argued that causation in general (which is direct or indirect) should be defined as the transitive closure of direct causation, as seems quite natural, though it is not unproblematic, and as many have assumed, though equally many have rejected it in the case of probabilistic causation.

Definition 7 modifies Hume's associationist theory of causation in three respects. First, it rests on a formally specified account of induction. Second, the

22 My opinion on this issue vacillates. In my (1980), I allowed for simultaneous causes, and in my other papers I didn't, or rather I avoided the issue. I have no philosophical principle for deciding it; therefore I make the stipulation which best serves my purpose at hand. So I do here, too; the reasons will become clear in section 5 below.

23 In the latter papers I did so only when simultaneity is excluded. The consequence of Definition 7 that in the case where $A$ and $B$ are simultaneous direct causation is symmetric is certainly forbidding. But in that case one might just read the definiendum in a different way, namely as saying that $A$ and $B$ are causally related without deciding which one is the cause and which one the effect. (Of course, nothing seems to able to decide this, and this is a most powerful argument for taking precedence strictly. But this issue is not our present concern.) And if one should find this last move unacceptable, then one should read this paper as being only about the restricted case where simultaneous variables are excluded. 
circumstances in which causal relations are situated are explicitly taken into account. This is done, at least implicitly, also by Hume, e.g. when he slips into counterfactual explanations of causation ${ }^{24}$, but he does not make a point of it. ${ }^{25}$ Third, the contiguity condition ${ }^{26}$, which would be reasonable only for direct causation, anyway, is dropped. As I explain more fully in (1990b), p. 129, the contiguity condition would force an unwantedly orderly behavior on subjectively relativized causation; we shall see that it comes into play only in the objectification of causation. ${ }^{27}$

I think that Definition 7 (and the just mentioned extension to indirect causation) is on the whole better able than other accounts to deal with intricate causal situations discussed in the literature. One problem to which we shall return is presented by cases of (symmetric) causal overdetermination which are usually exemplified by the firing squad (where several soldiers shoot the victim at the same time). Such cases are apparently possible. But they are a great mystery, if not an impossibility for all realistic accounts of causation; it seems that the only thing the realist can do is to explain them away (cf. Lewis (1986), pp.193-212). For Definition 7, by contrast, there is no mystery at all. The two overdetermining causes may be simply conceived as additional causes; each of the two is an additional cause of the effect in the presence of the other one.

There is a snag, however. The notion of an additional cause seems to make sense only within such an epistemically relativized framework and not within a realistic framework, because something true under some conditions cannot be more true under other conditions. On the one hand, the snag would explain and justify the difficulties of the realists in handling overdetermination. On the other hand, it accentuates the problem of objectification. Some causal relations seem to be objectifiable and others do not. But which ones are and which ones are not? And what, in the first place, could all that talk of objectification mean?

\footnotetext{
24 As he does in (1777), section VII, part 2.

25 Of course, the point has soon been acknowledged, e.g. by Mill (1843), book III, ch. V.

26 That is, the temporal part of it; spatial relations are here out of consideration.

27 Thus, the role Hume assigns to the contiguity condition indicates to me that he does not clearly separate subjective and objective aspects of causation.
} 


\section{An Explication of Objectification}

The having of a certain plain belief is a subjective affair; it applies to some epistemic subjects and does not apply to others. But the plain belief itself can be true or false, and its truth is an objective matter; it is as objective as truth is and in no way to be subjectively relativized. Formally: Let $\omega \in \Omega$ be the actual world. Then the plain belief in the proposition $A$ is true iff the proposition $A$ itself is true, i.e. iff $\alpha \in A$. Thus, plain beliefs have objective truth conditions; the truth condition of the plain belief in $A$ is just the proposition $A$ itself. These are trivial observations, deriving from the obvious one-one-correspondence between plain beliefs and the propositions which they are about. In the light of a lot of literature about the content of beliefs, this is at least implausible. ${ }^{28}$ But it is a direct consequence of my not worrying about these problems and burdening propositions with the double role as truth conditions and as contents of beliefs.

My objectification problem now is to which extent these observations apply also to other epistemic states and in particular to NCFs, to which extent other epistemic states and in particular NCFs may be said to be objectively true or false. This is less trivial, as is easy to see:

At first, one might say that NCFs have truth conditions as well. A NCF $\kappa$ has a net content, and the net content tells which propositions are plainly believed in $\kappa$. Thus, $\kappa$ may be said to be true iff all its plain beliefs are true, i.e. iff its net content is true, i.e. iff $\alpha \in \kappa^{-1}(0)$; and hence its truth condition is just its net content. However, this offers only very partial objectification. The correspondence between NCFs and their truth conditions in this sense is not one-one, but badly many-one, because a NCF's grading of disbelief below the net content may vary arbitrarily without affecting its truth condition. Thus, though objective truth has some selective power concerning NCFs, it affords no distinction among all the NCFs having the same truth condition, but differing wildly in their inductive behavior and other aspects.

These further observations teach us two things. First, when trying to objectify epistemic states, one has to refer to a certain feature or aspect of these states, and it is this feature on which objectification concentrates. In the last paragraph, the feature considered was the set of plain beliefs according to a NCF. But one may, and we shall, consider other features as well, e.g. the relation of direct causation

\footnotetext{
28 Indeed, the important insight of Kripke (1972) that a priori and (metaphysically) necessary truths are just two different kinds of things immediately implies that contents of beliefs and truth conditions also are two different kinds of things.
} 
according to a NCF.

Secondly, objectification may be only partial. It would be complete, if one had a one-one-correspondence between all the epistemic states considered and something objective like truth conditions. This may be impossible to reach, however. Usually, only epistemic states of a particular kind will yield to such a one-onecorrespondence, namely only those epistemic states which behave uniformly with respect to the feature considered. For example, if only belief functions $\beta$ are considered which behave uniformly with respect to plain belief, e.g. for which $\beta(A)=$ 1 for all $A$ with $\beta(A)>0$, these are easily put into a one-one-correpsondence with propositions. But then only such belief functions may be called objectifiable with respect to plain belief; and in this sense belief functions are only partially objectifiable with respect to plain belief.

Therefore, I propose the following explication of what it means to objectify NCFs. The first thing to do is to specify a natural association of propositions with features of NCFs. Here, a feature of NCFs is just any $n$-place relation $(n \geq 1)$ obtaining relative to a $\mathrm{NCF}$ (or, alternatively, any $n+1$-place relation the $n+1$ st place of which is taken by a NCF). The most natural association I can think of is then given by

Definition 8: Let $R$ be a NCF-relative $n$-place relation, and $x_{1}, \ldots, x_{n}$ be any objects in the domain of $R$. Then the proposition $E_{R}\left(x_{1}, \ldots, x_{n}\right)$ associated with $R\left(x_{1}, \ldots, x_{n}\right)$ is to be the strongest (i.e. smallest) proposition $A$ for which $R\left(x_{1}, \ldots, x_{n}\right)$ w.r.t. a $\mathrm{NCF} \kappa$ implies that $A$ is plainly believed in $\kappa$, i.e. which is such that for each NCF $\kappa, \kappa^{-1}(0) \subseteq A$ if $R\left(x_{1}, \ldots, x_{n}\right)$ w.r.t. $\kappa$.

The significance of this definition is best shown by examples; their claims may easily be seen to be correct:

(1a) $R(A)$ obtains relative to $\kappa$ iff $A$ is plainly believed in $\kappa$, then $E_{R}(A)=A$.

(1b) $R(A, B)$ obtains relative to $\kappa$ iff $B$ is plainly believed in $\kappa$ conditional on $A$, i.e. iff $\kappa(\sim B \mid A)>0$, then $E_{R}(A, B)=\sim A \cup B:=A \rightarrow B$, whereby $\rightarrow$ is defined as the set theoretical operation representing material implication for propositions. $^{29}$

(2a) $R(A, B)$ obtains relative to $\kappa$ iff $A$ is an additional reason for $B$ relative to $\kappa$,

29 I hope this notation is less misleading than helpful. 
then $E_{R}(A, B)=B$.

(2b) If $R(A, B)$ obtains relative to $\kappa$ iff $A$ is a sufficient reason for $B$ relative to $\kappa$, then $E_{R}(A, B)=A \rightarrow B$,

(2c) If $R(A, B)$ obtains relative to $\kappa$ iff $A$ is a necessary reason for $B$ relative to $\kappa$, then $E_{R}(A, B)=\sim A \rightarrow \sim B$.

(2d) If $R(A, B)$ obtains relative to $\kappa$ iff $A$ is a weak reason for $B$ relative to $\kappa$, then $E_{R}(A, B)=\sim B$.

(2e) If $R(A, B)$ obtains relative to $\kappa$ iff $A$ is a reason for $B$ relative to $\kappa$, then $E_{R}(A, B)=\Omega$.

(3a) If $R(A, B, \omega)$ obtains relative to $\kappa$ iff the $i$-proposition $A$ is an additional direct cause of the $j$-proposition $B$ in $\omega$ relative to $\kappa$, then $E_{R}(A, B, \omega)=$ $C_{A, B, \omega} \rightarrow B$.

(3b) If $R(A, B, \omega)$ obtains relative to $\kappa$ iff $A$ is a sufficient direct cause of $B$ in $\omega$ relative to $\kappa$, then $E_{R}(A, B, \omega)=C_{A, B, \omega} \cap A \rightarrow B$.

(3c) If $R(A, B, \omega)$ obtains relative to $\kappa$ iff $A$ is a necessary direct cause of $B$ in $\omega$ relative to $\kappa$, then $E_{R}(A, B, \omega)=C_{A, B, \omega} \cap \sim A \rightarrow \sim B$.

(3d) If $R(A, B, \omega)$ obtains relative to $\kappa$ iff $A$ is a weak direct cause of $B$ in $\omega$ relative to $\kappa$, then $E_{R}(A, B, \omega)=C_{A, B, \omega} \rightarrow \sim B$.

(3e) If $R(A, B, \omega)$ obtains relative to $\kappa$ iff $A$ is a direct cause of $B$ in $\omega$ relative to $\kappa$, then $E_{R}(A, B, \omega)=\Omega$.

So far, we have associated a proposition with a NCF-feature $R$ only as applied to certain items $x_{1}, \ldots, x_{n}$. But this is immediately extended to an association of a proposition with the NCF-feature itself:

Definition 9: Let $R$ be a NCF-relative $n$-place relation. Then the proposition $E_{R}(\kappa)$ associated with $R$ in relation to the NCF $\kappa$ is defined as the proposition $\bigcap\left\{E_{R}\left(x_{1}, \ldots, x_{n}\right) \mid R\left(x_{1}, \ldots, x_{n}\right)\right.$ obtains relative to $\left.\kappa\right\}$.

Together with Definition 8 this implies that $E_{R}(\kappa)$ is plainly believed in $\kappa$; indeed $E_{R}(\kappa)$ is the strongest proposition which is plainly believed in $\kappa$ in virtue of how the feature $R$ is realized in $\kappa$.

The second thing to do is to reverse the procedure and to inquire whether a $\mathrm{NCF} \kappa$ may be uniquely reconstructed from the proposition $E_{R}(\kappa)$ associated with 
the feature $R$; this allows, so to speak, to transfer the objectivity of $E_{R}(\kappa)$ to $\kappa$ itself. This unique reconstructibility seems also to be required for calling $\kappa$ to be objectifiable w.r.t. $R$. However, it seems feasible only when the feature $R$ is realized in $\kappa$ in some uniform way; I cannot imagine any way of encoding into $E_{R}(\kappa)$ different ways of realizing $R$. Therefore we need to refer to such a uniform specification of $R$. Moreover, we shall see that the reconstruction may work only under certain conditions; therefore we also need to refer to such conditions. Now I am finally prepared to offer my explication of objectifiability:

Definition 10: Let $S$ be any specification of the feature $R$; this means that, for each NCF $\kappa, R\left(x_{1}, \ldots, x_{n}\right)$ obtains relative to $\kappa$, if $S\left(x_{1}, \ldots, x_{n}\right)$ obtains relative to $\kappa$; and let $F$ be any condition on the items in the field of $R$. Then a NCF $\kappa$ is objectifiable with respect to $R$ (or, $\kappa$ is an objectification of $R$ ) under the specification $S$ given condition $F$ iff, given $E=E_{R}(\kappa), \kappa$ is the only NCF such that the following holds:

(a) for all $x_{1}, \ldots, x_{n}, R\left(x_{1}, \ldots, x_{n}\right)$ obtains relative to $\kappa$ if and only if $S\left(x_{1}, \ldots, x_{n}\right)$ obtains relative to $\kappa$,

(b) for all $x_{1}, \ldots, x_{n}, S\left(x_{1}, \ldots, x_{n}\right)$ obtains relative to $\kappa$ if and only if $x_{1}, \ldots, x_{n}$ satisfy the condition $F$ and $E \subseteq E_{R}\left(x_{1}, \ldots, x_{n}\right)$.

Moreover, we omit reference to the condition $F$ iff $F$ is empty, and we omit reference to the specification $S$ by existential quantification.

Thus I slip into two ways of talking. Sometimes I say that a NCF is objectifiable w.r.t to some feature, and sometimes I say that that feature itself is objectifiable. Both ways of talking seem appropriate, though they may be a bit confusing.

Clause (a) expresses the uniform realization of the feature $R$ in the NCF $\kappa$; it requires $R$ to be realized in $\kappa$ only in the way $S$. Clause (b) says that the relation $R$, as it obtains relative to $\kappa$, is determined by the condition $F$ and by $E_{R}(\kappa)$. And the uniqueness clause guarantees that $\kappa$ may be uniquelly reconstructed from the information specified in (a) and (b).

Unconditional objectifiability means that one can infer from $E_{R}(\kappa)$ alone for which $x_{1}, \ldots, x_{n}$ the feature $R$ holds relative to $\kappa$. But it may be that the condition $F$ is needed for this inference. Of course, one may find conditions which trivialize conditional objectifiability; how objective conditional objectification really is depends on how objective the condition $F$ is.

Again, all this is too abstract to assess its significance. So let's return to the examples (1)-(3) already introduced and investigate the extent to which they allow objectification. 


\section{The Objectification of Induction and Causation}

(1a) Let $R(A)$ obtain relative to $\kappa$ iff $A \neq \Omega$ and $A$ is plainly believed in $\kappa$, and for some positive integer $m$, let $S(A)$ obtain relative to $\kappa$ iff $A \neq \Omega$ and $\beta(A)=$ $\kappa(\sim A)=m .{ }^{30}$ Then $\kappa$ is an objectification of $R$ under $S$ iff, for all $\omega \in \Omega, \kappa(\omega)=$ $m$, if $\kappa(\omega)>0$. In short, plain belief is unconditionally objectifiable.

This is also intuitively plausible; a NCF is uniquely reconstructible from what is plainly believed in it only if it does not differentiate among the disbelieved worlds. Note, however, that such NCFs are unfit epistemic states. Whenever you are in such a state and accept a piece of information contradicting your plain beliefs (this is quite common), you then believe only the information accepted and nothing more; and this is a devastatingly cautious inductive behavior.

(1b) Let $R(A, B)$ obtain relative to $\kappa$ iff $A$ does not logically imply $B$ and $\kappa(\sim B$ । $A)>0$, i.e. $B$ is plainly believed in $\kappa$ conditional on $A$; and for some integer $m$, let $S(A, B)$ obtain relative to $\kappa$ iff $A$ is not a subset of $B$ and $\kappa(\sim B \mid A)=m$. Then $R$ is objectified under $S$ by the very same NCF as in (1a). ${ }^{31}$ There are thus only poor unconditional objectifications of conditional plain belief because the objectifying NCFs of (1a) have only one genuine level of conditionality. By this I mean that conditional on something disbelieved only that condition and its logical consequences are believed according to these NCFs. Of course, this is tantamount to their unfit inductive behavior just mentioned.

(2) Here the results are even more negative:

(2a,d) If $R(A, B)$ means that $A$ is an additional reason for $B$ w.r.t. $\kappa$, there is no unconditional objectification for $R$, for the simple reason that, since $E_{R}(A, B)=B$, there is no way of telling from $E_{R}(\kappa)$ whether $A$ or $\sim A$ is to be an additional reason for $B$. The same applies to weak reasons, because $A$ is a weak reason for $B$ iff $\sim A$ is an additional reason for $\sim B$.

(2e) A fortiori, there is no objectification at all for the relation of being a reason simpliciter.

$(2 \mathrm{~b}, \mathrm{c})$ If $R(A, B)$ means that $A$ is a sufficient reason for $B, R$ can still not be objectified unconditionally. In this case we have $E_{R}(A, B)=A \rightarrow B$. So, if $\kappa$ is to be objectifiable w.r.t. $R, A$ would have to be a sufficient reason for $B$ according to $\kappa$ if and only if $E_{R}(\kappa) \subseteq A \rightarrow B$. The set of sufficient reasons for $B$ according to $\kappa$ would therefore have to be a (complete) ideal, i.e. it would have to be non-empty,

$30 A=\Omega$ must be excepted because $\beta(\Omega)=\kappa(\varnothing)=\infty \neq m$ for any $m$.

31 For proof consider a NCF $\kappa$ taking more than the two values 0 and $m$. Then it is easily seen that $\kappa$ contains conditional beliefs of differing strength and cannot uniformly realize $R$ as $S$. 
it would have to be closed under (arbitrary, not only finite) union, and it would have to contain all the subsets of its members. ${ }^{32}$ But usually there are many propositions $B$ for which this set is not an ideal.

Let's be more precise. First, the special role of $\varnothing$ must be observed. $\varnothing$ is never a reason for $B$, but $E_{R}(\kappa) \subseteq \varnothing \rightarrow B=\Omega$ always holds. However, there is no problem in making special provisos concerning $\varnothing$. The problem is rather this: Suppose that $A$ is a sufficient reason for $B$ w.r.t. $\kappa$. It is easily seen that this is the case if and only if $\kappa(A \cap B)<\kappa(A \cap \sim B)$ and $\kappa(\sim A \cap B) \geq \kappa(\sim A \cap \sim B)$. Thus a subset $A^{\prime}$ of $A$ which is not a sufficient reason for $B$ may be constructed simply by deleting from $A \cap B$ sufficiently many worlds with low $\kappa$-values and putting them into $\sim A^{\prime} \cap B$. If this construction works, the set of sufficient reasons for $B$ is not an ideal; the construction works for at least some reasons $A$ whenever for each $v \in$ $\sim B$ there is a $\omega \in B$ such that $\kappa(v) \leq \kappa(\omega)$; and there are such propositions $B$ whenever there are at least two worlds receiving (not necessarily different) nonzero $\kappa$-values.

Since $A$ is a necessary reason for $B$ iff $\sim A$ is a sufficient reason for $\sim B$, the very same observations hold also for the relation of being a necessary reason.

Compare this with the case (1b) of conditional plain belief. There, objectification also requires that the set of conditions under which a proposition $B$ is plainly believed is a (complete) ideal. This is indeed the case for the NCFs specified there, i.e. for the NCFs taking only two values (though for all other NCFs conditional belief is non-monotonous in the sense that strengthening the condition need not preserve conditional belief). However, this tiny success does not carry over to sufficient reasons, because being a sufficient reason consists in conditional belief plus no belief under the contrary condition.

The general problem is to reconstruct the (sufficient) reason relation $R$ from all the material implications entailed by $E_{R}(\kappa)$; and it seems that there is no general solution. Perhaps the device of conditional objectification helps. But what should the condition $F$ be which selects the sufficient reasons from all these material implications? There is a trivial answer: let $F$ itself be the sufficient reason relation according to $\kappa$. It was this answer which I had in mind when emphasizing that the objectivity reached by conditional objectification depends on the objectivity of the condition itself. This trivial condition is as subjective as the NCF $\kappa$ referred to; and I do not see any general, more objective criterion which affords a successful selection.

32 Equivalently, a complete ideal of propositions is just the set of all the negations of the members of a deductively closed set of propositions. 
(3) Matters look much better, however, in the case of causal relations because in this case the relevant material implications are severely restricted in form. So let us return to our initial topic, the objectification of causation. The first assertion is negative: Additional direct causes (3a), weak direct causes ( $3 \mathrm{~d})$, and thus direct causes in general (3e) can obviously be as little objectified as additional and weak reasons. Still, this observation explains why additional and weak causes have been totally neglected in the literature and in particular why causal overdetermination is such a problem for the realist and cannot be accounted for within a realistic setting in the way indicated at the end of section 2 . There are more positive news, however, in the remaining cases:

$(3 \mathrm{~b}, \mathrm{c})$ Let $R(A, B, \omega)$ obtain relative to $\kappa$ iff $A$ is a sufficient direct cause of $B$ in $\omega$ relative to $\kappa . E_{R}(\kappa)$ may then be called the causal law of $\kappa$.

Note that necessary causation may be defined by sufficient causation: $A$ is a necessary direct cause of $B$ in $\omega$ iff $\sim A$ is a sufficient direct cause of $B$ in a suitable variation $\omega^{\prime}$ of $\omega$ concerning the variables $A$ and $B$ are about. Thus, whatever the objectification of sufficient direct causation, it is also an objectification of necessary direct causation to the very same extent; in particular, starting from necessary direct causation we would have arrived at the very same causal law. This is the reason why the following considerations may be restricted to sufficient causation.

When trying to objectify $R$, we must again refer to some uniform specification $S$ of $R$. There is obviously only one way of doing so: for some positive integer $m$, let $S(A, B, \omega)$ obtain relative to $\kappa$ iff $A$ is an $i$-proposition, $B$ a $j$-proposition, $i \leq j$, $\omega \in \Omega$, and $\kappa\left(\sim B \mid A \cap C_{A, B, \omega}\right)=m$ and $\kappa\left(\sim B \mid \sim A \cap C_{A, B, \omega}\right)=0$.

In order to objectify sufficient causation we have to rediscover appropriate NCFs from causal laws in some unique way. Thus, the first step is to find a general association of potential causal laws, which, objectively, are just true or false propositions, with NCFs giving these propositions a causal interpretation. The only feasible association I have found is this:

As a piece of notation, define first, for each world $\omega$ and each $J \subseteq I, \omega_{j}=\{v \mid$ $\mathrm{v}(i)=\omega(i)$ for all $i \in J\}$ as the proposition that the variables in $J$ behave as they do in $\omega$; in particular define $\omega_{j}=\omega_{\{j\}}$ as the proposition that $j$ takes the value in $\omega, \omega_{<j}=\{\mathrm{v} \mid \mathrm{v}(i)=\omega(i)$ for all $i<j\}$ as the proposition that the past of $j$ is as it is in $\omega$, and similarly $\omega_{\leq j}$ and $\omega_{\approx j}$; moreover define $I_{<j}=\{i \in I \mid i<j\}$, and similarly $I_{\leq j}$ and $I_{\approx j}$.

With respect to any proposition $L, C$ is called a $L$-sufficient condition of the $j$ proposition $B$ iff $C$ is a $I_{\leq j}-\{j\}$-proposition (i.e. about the past and presence of $B$ ) 
and $L \subseteq C \rightarrow B$; the L-sufficient condition $S C_{L}(B)$ of $B$ is defined as the union of all its $L$-sufficient conditions (i.e. as the largest or weakest $I_{\leq j}-\{j\}$-proposition $C$ such that $L$ entails $C \rightarrow B$ ). We define a proposition $L$ to be a law of succession iff for each variable $j \in I$ and $j$-proposition $B \neq \Omega S C_{L}(B)$ is a $I_{<j}$-proposition (only about the past of $B$ ) and there is an $I_{\approx i}$-proposition $A \neq \Omega, i$ being a variable immediately preceding $j$ (if $j$ is the temporally first variable; $I_{\approx i}=\varnothing$ ), such that $S C_{L}(B)=A \cup S C_{L}(A)$. In that case, there is exactly one such $I_{\approx i}$ proposition $A$, which will be called the immediate L-sufficient condition of $B$ and denoted $I S C_{L}(B)$. Of course, we usually have $I S C_{L}(B) \neq S C_{L}(B)$ because whatever is sufficient for $I S C_{L}(B)$ is also sufficient for $B$. Intuitively, a law of succession is just a conjunction of material implications of the form "if now $A$, then next $B$ " which, however, does not entail any categorical proposition about a single variable (but see the qualification in condition (II') below).

Let us say that $L$ is violated by the variable $j$ in the world $\omega$ iff the $L$-sufficient condition of $\sim \omega_{j}$ obtains in $\omega$, i.e. iff $\omega \in I S C_{L}\left(\sim \omega_{j}\right)$. Now we can finally specify the NCF $\kappa_{L}$ associated with the law $L$ of succession by defining $\kappa_{L}(\omega)=r \cdot m$, where $r$ is the number of variables by which $L$ is violated in $\omega$ ( $r$ is finite because $I$ was assumed to be finite).

The $\kappa_{L}$ 's so defined do not have a wholly satisfying inductive behavior, but they are much more reasonable than those objectifying (conditional) plain belief. The reason is that there may occur as many violations of $L$ as there are variables and that $\kappa_{L}$ may thus take as many different values. The predictive and retrodictive behavior of these NCFs under various conditionalizations may therefore be complex; but it is characterized by two simple properties: First, simultaneous variables are always independent conditional on their past (i.e. on the set of variables preceding them), as is easily verified. Secondly, any $j$-proposition is expected to obtain whenever its immediate $L$-sufficient condition is satisfied. Hence, such a $\kappa_{L}$ has a peculiar inductive rigidity: However often $L$ is violated, $\kappa_{L}$ is not deterred from inductive inferences, but expects invariably that no further violations of $L$ will occur.

The $\kappa_{L}$ 's are my only candidates for objectifying sufficient causation. Are they suited for that purpose? Not generally; there are two problems entailing substantial, though instructive restrictions.

The first problem is that $L$ need not be the causal law of $\kappa_{L}$. We can show that 
$L \subseteq E_{R}\left(\kappa_{L}\right)^{33}$, but the converse does not necessarily hold. Suppose, e.g., that there are just three variables $i \approx j<k$, that for some $\omega A=\omega_{i}, B=\omega_{j}$, and $C=\omega_{k}$, and that $L=A \cup B \rightarrow C$. There are then no sufficient causes of $C$ in $\omega$ w.r.t. $\kappa_{L}$ (even though $\omega_{<k}$ is a $L$-sufficient condition of $C$ ); in fact, there are only two ways for $R$ to obtain relative to $\kappa_{L}$ : we have $R\left(A, C, \omega^{\prime}\right)$, where $\left\{\omega^{\prime}\right\}=A \cap \sim B \cap C$, and $R(B$, $\left.C, \omega^{\prime}\right)$, where $\left\{\omega^{\prime}\right\}=\sim A \cap B \cap C$. Hence, $E_{R}\left(\kappa_{L}\right)=(A \cap \sim B) \cup(\sim A \cap B) \rightarrow C \neq$ $L$.

But surely, the identity of $L$ and $E_{R}\left(\kappa_{L}\right)$ is required for a unique association of NCFs with causal laws. So, when does this identity hold? This is answered by a theorem:

$E_{R}\left(\kappa_{L}\right)=L$ holds if and only if the following two conditions are satisfied:

(I) each $j$-proposition $B \neq \Omega(j \in I)$ has at least one sufficient direct cause in each $\omega \in I S C_{L}(B)$ w.r.t. $\kappa_{L}$, and

(II) $L=\bigcap\left\{S C_{L}(B) \rightarrow B \mid B\right.$ is a $j$-proposition for some $\left.j \in I\right\}=\bigcap\left\{I S C_{L}(B) \rightarrow\right.$ $B \mid B$ is a $j$-proposition for some $j \in I$ \}

where these conditions are, respectively, equivalent to

(I') for each $j \in I, j$-proposition $B$, and $\omega \in I S C_{L}(B)$ the intersection of all subsets $J$ of $I_{\approx i}$ with $\omega_{J} \subseteq I S C_{L}(B)$ (where $i$ is some variable immediately proceeding $j$ ) is non-empty, and

(II') if $D_{\omega_{j}}$ is defined as the smallest or strongest $I_{\approx j}$-proposition such that $L \subseteq$ $\omega_{<j} \rightarrow D_{\omega_{j}}$ then $D_{\omega_{j}}$ is purely conjunctive for each $\omega \in \Omega$ and $j \in I$, i.e. if $I_{\approx j}$ $=\left\{i_{1}, \ldots, i_{s}\right\}$, there are $i_{r}$-propositions $A_{r}(r=1, \ldots, s)$ such that $D_{\omega_{j}}=A_{1} \cap$ $\ldots \cap A_{s}$.

Proof (and remarks): The second equation of (II) always holds for laws of succession; and the equivalence of the first equation of (II) with (II) is easily proved. I have mentioned (II') only because it makes the content of (II) more perspicuos.

Concerning the equivalence of (I) and (I') note that whenever the $i$-proposition $A$ is a sufficient cause of $B$ in $\omega$ w.r.t. $\kappa_{L}$, so is $\omega_{i}$. Then it is easily seen that $\omega_{i}$ is a sufficient cause of $B$ in $\omega$ w.r.t. $\kappa_{L}$ if and only if $i$ is a member of each subset $J$

33 Proof: Let $A$ be a sufficient cause of the $j$-proposition $B$ in $\omega$ w.r.t. $\kappa_{L}$. This implies that $L$ is violated by $j$ in all worlds $v$ with $v \in A, C_{A, B, v}=C_{A B, \omega}$, and $v \in \sim B$, i.e. that $A \cap C_{A, B, \omega}$, is a $L$ sufficient condition of $B$. Since this holds for all $A, B$, and $\omega$, we have $L \subseteq E_{R}\left(\kappa_{L}\right)$. 
of $J_{\approx i}$ with $\omega_{J} \subseteq I S C_{L}(B)$. (I) is, of course, a version of the principle of causality. It is at least interesting that it is required at this stage of the argument (though I am not sure whether the reason for this lies only in my formalization). The point of the equivalent condition (I') is to show that (I), though it seems to be about causation and thus about $\kappa_{L}$, is in fact a condition solely about the logical form of the law of succession $L$, and clearly, (II) is so, too.

Now to the main point of the theorem. In view of the observations that, if there is a sufficient direct cause of $B$ in $\omega$, then for some $i \omega_{i}$ is a sufficient direct cause of $B$ in $\omega$ and that $B$ can have sufficient direct causes only in the worlds in $I S C_{L}(B)$, (I) implies that $E_{R}\left(\kappa_{L}\right)=\bigcap\left\{A \cap C_{A, B, \omega} \rightarrow B \mid R(A, B, \omega)\right.$ obtains w.r.t. $\left.\kappa_{L}\right\}=\bigcap\left(I S C_{L}(B) \rightarrow B \mid\right.$ is a $j$-proposition for some $\left.j \in I\right\}$ and (II) ensures that the latter term is equal to $L$. Conversely, suppose that (I) holds, but (II) doesn't. Thus, some $D_{\omega_{j}}$ of (II') are not of the conjunctive form described there, but the $D_{\omega_{j}}$ defined w.r.t. $E_{R}\left(\kappa_{L}\right)=\bigcap\left\{I S C_{L}(B) \rightarrow B \mid B\right.$ is a $j$-proposition for some $\left.j \in I\right\}$ always are. Hence, $E_{R}\left(\kappa_{L}\right) \neq L$ in this case. Or suppose that (I) does not hold for a certain $\omega \in I S C_{L}(B)$ and $j$-proposition $B$. Then $E_{R}\left(\kappa_{L}\right) \subseteq \omega_{<j} \rightarrow B$ does not hold in virtue of $\omega$, and there is no other causal relationship bringing about this inclusion. But $L \subseteq \omega_{<j} \rightarrow B$, and hence $E_{R}\left(\kappa_{L}\right) \neq L$ also in this case.

This theorem provides provides a satisfying solution of the first problem of objectification. But there is a second and more substantial problem, namely that the causal relations obtaining relative to NDF $\kappa$ can generally not be rediscovered from its causal law. The reason is that the causal law is unable to distinguish the following two causal situations:
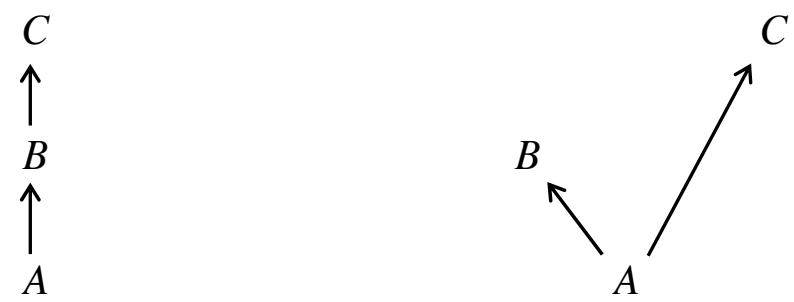

where $A$ precedes $B, B$ precedes $C$, and $A$ is a necessary and sufficient direct cause of $B$ and where there is a causal chain from $A$ over $B$ to $C$ in the one case and a causal bifurcation in the other. ${ }^{34}$ This is a grave and basic problem for all

34 It is instructive to construct two NCFs generating the different causal situations and to see the difference vanish in the causal law which is one and the same for the two NCFs. 
realistic theories of deterministic causation. ${ }^{35}$ The only natural way I see to get rid of this problem is to assume that direct causes immediately precede their direct effects. ${ }^{36}$ This assumption makes the case of a causal bifurcation impossible when $B$ precedes $C$ and allows for it only when $B$ and $C$ are simultaneous; and it does away with the unclear relations between simultaneous variables. Thus it enables us to infer the causal relations from a causal law. Intuitively it is clear that I have already built in his assumption into our account by deriving the $\kappa_{L}$ 's from laws $L$ of succession in the way it did.

Note that this assumption to which objectification will be conditioned is a perfectly objective one about how causation relates to time; it does not refer to epistemic states or other subjective matters. The assumption is not really necessary for objectification. Presumably, any other assumption fixing the relation between time and causation (e.g. that each variable directly affects only the second next variable) would do as well. But this would be utterly artificial; contiguity is certainly the most natural thing to assume. Note further that, if I am right, it is at this point of objectification where the (temporal) contiguity condition comes into play and not already in the definition of causation, as Hume thought.

So, let's bring home the point in a precise way. First, there is a little problem with propositions about temporally first variables. Since these propositions do not have causes, causal relations cannot fix any belief values for them, in fact, these belief values may vary arbitrarily in a NCF without changing the causal relations concerning later variables. For the same reason, however, it is desirable that a NCF $\kappa$ objectifying sufficient causation does not have any opinion on these propositions, i.e. that $\kappa(B)=0$ for each non-empty proposition $B$ about the first variables. In order to attain this desired result, I propose to apply a technical trick and slightly extend the relations $R$ and $S$ so that they cover also this problematic case: Define that $R^{\prime}(A, B, \omega)$ obtains relative to $\kappa$ iff $R(A, B, \omega)$ obtains relative to $\kappa$ or $\omega \in A \cap B, B$ is about a temporally first variable, $\mathrm{A}=\Omega$, and $\kappa(\sim B)>0$, and that $\mathrm{S}^{\prime}(A, B, \omega)$ obtains relative to $\kappa$ iff $S(A, B, \omega)$ obtains relative to $\kappa$ or $\omega \in A \cap$ $B, B$ is about a first variable, $A=\Omega$ and $\kappa(\sim B)=m$.

Now let's state a condition on the relata of direct causation:

(III) $A, B$, and $\omega$ are such that $A$ is a $i$-proposition for some $i \in I$ immediately preceding $j$ and $B$ a $j$-proposition.

35 Cf., e.g., Mackie (1974), pp. $81 \mathrm{ff}$.

36 Recall that time was assumed to be discrete. Thus it makes sense to talk of immediate temporal precedence. 
Then we have the desired theorem: The NCF $\kappa$ is an objectification of $R^{\prime}$ under the specification $S^{\prime}$ given condition (III) if and only if $\kappa=\kappa_{L}$ for some law $L$ of succession satisfying (I) and (II).

Proof (and remarks): Let $L$ be a law of succession. Trivially, $\kappa_{L}$ satisfies clause (a) of Definition 10. It is also clear from the definition of laws of succession and from the construction of $\kappa_{L}$ that $\kappa_{L}$ satisfies clause (b) of Definition 10, provided $L$ conforms to (I) and (II). Note that we could not do without condition (III) at this point. For, we may have $L \subseteq A \rightarrow B, L \subseteq B \rightarrow C$ and thus also $L \subseteq A$ $\rightarrow C$. But the latter material implication is not to represent a direct causal relationship; and this is excluded by condition (III).

In order to see that $\kappa_{L}$ is the only NCF satisfying (a) and (b), note first the simultaneous variables are bound to be independent conditional on their past w.r.t. to any NCF $\kappa$ satisfying (b); this follows from the fact that according to (b) $R(A$, $B, \omega)$ is not to obtain w.r.t. $\kappa$ for all $\omega \in \Omega$ and all simultaneous $A$ and $B$. Next observe that for any NCF $\kappa$ satisfying (a) and (b) the value $\kappa\left(\omega_{j} \mid \omega_{<j}\right)$ is thereby determined for each $\omega \in \Omega$ and $j \in I$ to be equal to $\kappa_{L}\left(\omega_{j} \mid \omega_{<j}\right) \quad(=m$ or 0 , depending on whether $\omega_{<j} \subseteq I S C_{L}\left(\sim \omega_{j}\right)$ or not $)$. But $\kappa$ is uniquely determined by all these values since, given the independence of simultaneous variables conditional on their past, $\kappa(\omega)=\Sigma_{j \in I} \kappa\left(\omega_{j} \mid \omega_{<j}\right)$. Thus $\kappa$ is $\kappa_{L}$.

It is in this reasoning, by the way, where the liberal version of Definition 7 admitting simultaneous causation is technically required. If we would have ruled out simultaneous causation by definition, then no condition whatsoever on causal relations could say anything about simultaneous variables and could force on them the conditional independence needed for uniquely reconstructing $\kappa_{L}$ from the causal relationship obtaining relative to it.

Now suppose conversely that $\kappa$ is the unique NCF satisfying clause (a) and (b) of Definition 10. It is easily seen that (b) entails entails that $E_{R^{\prime}}(\kappa)$ is a law of succession satisfying (I) and (II). And then it is clear that (a) forces $\kappa$ to be of the special form $\kappa_{L}$, where $L=E_{R^{\prime}}(\kappa)$.

Thus, we have finally arrived at an answer to the question to which extent sufficient and/or necessary direct causation is objectifiable. I do not know whether there are other plausible answers to that question within the framework I have been using. Within a richer framework, however, including, e.g., spatial considerations richer results would certainly be forthcoming.

To resume, what is a causal law? The answer is quite trivial. It is a proposition which can be true or false and which has a special form, namely that of a law of 
succession. This is certainly no news. So why deduce this familiar view in such a complicated fashion? In order to answer the question how such a proposition becomes a causal law. For, where does the causal role of such a proposition come from? From the above objectification, from uniquely associating with such a proposition a particular inductive behavior which is encoded in the corresponding objectifiable NCF and which interprets certain material implications entailed by that proposition as causal relations. This is my construal of the claim that causal laws are objectifications of inductive schemes.

Let me add a philosophical comment. The results obtained may be used as a very partial response to what might be called Quine's challenge. Quine's challenge is to explain how we can scientifically entertain our familiar intensional and intentional talk of beliefs, meanings, dispositions, causes ${ }^{37}$, etc.; in his terms, it is to reconstruct the second grade vernacular within the austere canonical notation of science which is, in effect, extensional first order predicate logic. This is a challenge, of course, because Quine has so forcefully argued over and over again that it cannot be met. And it seems that he is right; if we take the strict standpoint, the loose talk is lost forever.

But then it seems wrong to insist on the strict standpoint from which the rest is just unacceptable. A more fruitful strategy is to start from broader grounds including all kinds of non-extensional phrases, to characterize the virtues of extensional scientific language, and to describe the conditions under which these virtues can be realized. This is more fruitful because it allows us to get a theoretical hold on the relation between extensional and intensional talk, between our broader picture imbued with subjectivity and our attempts to reach scientific objectivity. In a way, the point of this paper is to show in one particular corner how such a theoretical hold may be constructively gained by specifying the conditions under which intensional causal conditionals reduce to extensional material implication, i.e. under which causation is objectifiable.

Putnam has also been impressed by Quine's arguments; but he draws a different conclusion, namely that the austere standpoint as Quine describes it is not an ideal, but an unattainable chimera. ${ }^{38}$ But this conclusion seems to overshoot the mark. A theory of objectification as it is envisaged here should show the extent to which the ideal is attainable. And it partially confirms Putnam. As we have seen, the notion of (deductive or inductive) reason is not objectifiable; and if this holds

37 Though Quine rather discusses dispositions, he explicitly assigns causation the same low status; cf., e.g., Quine (1969), pp. 132f.

38 Cf., e.g., Putnam (1983b). 
for our narrower and more technical notion, it holds all the more for Putnam's much broader and more sweeping notion of reason. But it also contradicts him. What is true of reason, need not, and does not, carry over to causation.

\section{Outlook}

As I have mentioned in the introduction, there is a far-reaching analogy between the deterministic case with which I have dealt here and the probabilistic case which is more familiar in some respects. It would therefore be most appealing to extend this analogy also to the topic of objectification and to look which grip one thereby gets on the notion of objective probability. Intuitively, the analogy is quite obvious. Sufficient causation is tantamount to the idea of determination, and similarly objective probability is tantamount to the idea of partial determination; the fact that some possible event has, immediately before its time of occurrence, some degree of chance means that it is only partially determined and that it is in no way further determinable. We arrived at laws of succession when objectifying sufficient causation; thus Markow processes, which obviously are the probabilistic counterparts of laws of succession and which always were the favorites for probabilistic models of causal processes, may similarly be crucial for understanding objective probability. And so on. But however suggestive such hints are, they clearly need to be worked out in detail. It would then be particularly interesting to compare the resulting account of the relation between subjective and objective probability with other accounts of which I have found Lewis (1980) and Skyrms (1984), ch. 3, to be most illuminating; I suspect that the similarities by far outweigh the differences.

The considerations presented make only a first step. As I have mentioned, the NCF's which are objectifiable in some respect are not very recommendable inductive schemes (and some are worse than others). Likewise on the probabilistic side, Bernoulli measures, for instance, according to which the variables considered are independent (and identically distributed) are bad inductive guides. Thus, the necessary second step is to follow de Finetti's strategy, i.e. to inquire the following questions. Which epistemic states are mixtures of objectifiable epistemic states? Which epistemic states can be uniquely represented as mixtures of objectifiable epistemic states? And which of these mixtures converge with increasing evidence to some objectifiable epistemic state?

There are powerful positive results in the probabilistic case: de Finetti's origi- 
nal theorem, a de Finetti-type theorem for Markov chains (proved in Diaconis, Freedman, 1980), and, as the strongest of all, the ergodic theorem (cf. Skyrms, 1984, ch. 3). The concept of a mixture makes sense also for NCFs; it is easily specified how to mix the NCFs in a given set by a NCF defined on that set so that the mixture is again a NCF. But, of course, there do not exist answers to the questions in terms of NCFs.

\section{References}

Aronson, J.L. (1971), “On the Grammar of 'Cause”, Synthese 22, 414-430.

Beauchamp, T., A. Rosenberg (1981), Hume and the Problem of Causation, Oxford University Press.

Carnap, R. (1966), Philosophical Foundations of Physics, Basic Books, New York.

Diaconis, P., D. Freedman (1980), “De Finetti's Theorem for Markov Chains”, Annals of Probability 8, 115-130.

Fair, D. (1979), “Causation and the Flow of Energy”, Erkenntnis 14, 219-250.

van Fraassen, B.C. (1989), Laws and Symmetry, Oxford University Press.

Gärdenfors, P. (1988), Knowledge in Flux, M.I.T. Press, Cambridge, Mass.

Hansson, S.O. (1990), “The Logic of Repeated Belief Changes”, forthcoming.

Hempel. C.G. (1965), Aspects of Scientific Explanation and Other Essays in the Philosophy of Science, Free Press, New York.

Hume, D. (1739), A Treatise Concerning Human Nature. Page numbers refer to the 2nd edition by P.H. Nidditch of the edition by L.A. Selby-Bigge, Oxford University Press 1978.

Hume, D. (1777), An Enquiry Concerning Human Understanding.

Kripke, S. (1972), "Naming and Necessity”, in: D. Davidson, G. Harman (eds.), Semantics of Natural Language, Reidel, Dordrecht, pp. 253-355.

Lewis, D. (1973), “Causation”, Journal of Philosophy 70, 556-567; also in Lewis (1986), pp. 159172 , supplemented by postscripts.

Lewis, D. (1980), “A Subjectivist's Guide to Objective Chance”, in: R.C. Jeffrey (ed.), Studies in Inductive Logic and Probability, vol. II, University of California Press, pp. 263-293; also in Lewis (1986), pp. 83-113, supplemented by postscripts.

Lewis, D. (1986), Philosophical Papers, vol. II, Oxford University Press.

Mackie, J.L. (1974), The Cement of Universe, Oxford University Press.

Mill, J.St. (1843), System of Logic.

Mühlhölzer, F. (1988), “On Objectivity”, Erkenntnis 28, 185-230. 
Putnam, H. (1983a), “Why There Isn't a Ready-Made World”, in: H. Putnam, Realism and Reason, Philosophical Papers, vol. 3, Cambridge University Press, ch. 12.

Putnam, H. (1983b), "Why Reason Can't Be Naturalized”, in: H. Putnam, Realism and Reason, Philosophical Papers, vol. 3, Cambridge University Press, ch. 13.

Quine, W.V.O. (1969), "Natural Kinds", in: W.V.O. Quine, Ontological Relativity and Other Essays, Columbia University Press, New York, ch. 5.

Salmon, W.C. (1984), Scientific Explanation and the Causal Structure of the World, Princeton University Press.

Shenoy, P. (1989), “On Spohn's Rule for Revision of Beliefs”, School of Business Working Paper No. 213, University of Kansas, Lawrence.

Skyrms, B. (1984), Pragmatics and Empiricism, Yale University Press.

Spohn, W. (1980), "Stochastic Independence, Causal Independence, and Shieldability", Journal of Philosophical Logic 9, 73-99.

Spohn, W. (1983), "Deterministic and Probabilistic Reasons and Causes", in: C.G. Hempel, H. Putnam, W.K. Essler (eds.), Methodology, Epistemology, and Philosophy of Science. Essays in Honour of Wolfgang Stegmüller on the Occasion of his 60th Birthday, Reidel, Dordrecht, pp. 371-396.

Spohn, W. (1988), "Ordinal Conditional Functions. A Dynamic Theory of Epistemic States", in: W.L. Harper, B. Skyrms (eds.), Causation in Decision, Belief Change, and Statistics, vol. II, Kluwer, Dordrecht, pp. 105-134.

Spohn, W. (1990a), “A General Non-Probabilistic Theory of Inductive Reasoning”, in: R.D. Shachter, T.S. Levitt, J. Lemmer, L.N. Kanal (eds.), Uncertainty in Artificial Intelligence 4, Elsevier, Amsterdam, pp. 149-158.

Spohn, W. (1990b), “Direct and Indirect Causes”, Topoi 9, 125-145.

Spohn, W. (1993), „Was ist eine Theorie der Rationalität?“, in: L.H. Eckensberger, U. Gähde (eds.), Empirische versus begriffliche Analysen in Fragestellungen der angewandten Ethik, Suhrkamp, Frankfurt a.M., to appear.

Tooley, M. (1987), Causation, Oxford University Press. 